\title{
A UTILIZAÇÃO DA Garcinia cambogia COMO COADJUVANTE NO TRATAMENTO DA OBESIDADE
}

\author{
THE USE OF Garcinia cambogia AS A CO-ADJUVANT WITHOUT TREATMENT OF \\ OBESITY
}

VERBINEN, $A^{1^{*}} ;$ OLIVEIRA, V. $B^{2}$

1 - Acadêmica do Centro Universitário Campos de Andrade (UNIANDRADE), Curitiba - PR, Brasil. 2 - Professor do Centro Universitário Campos de Andrade (UNIANDRADE), Curitiba - PR, Brasil. Autor para correspondência: e-mail: andressaverbi@hotmail.com

\section{RESUMO:}

A partir de dados da OMS (Organização Mundial de Saúde), a obesidade é entre as morbidades uma das mais preocupantes atualmente, visto que essa ainda pode ser precursora de outras doenças. Seu tratamento é de extrema importância, portanto levandoo em consideração, esse trabalho tem como objetivo verificar através de uma revisão bibliográfica a utilização da espécie vegetal Garcinia cambogia, como sendo coadjuvante no tratamento da obesidade. A obesidade dispõe de tratamentos voltados a reeducação alimentar e prática de atividade física como tratamentos base, porém a $G$. cambogia apresenta-se como um fitoterápico de suma importância, ainda salientando seus benefícios para com resultados visíveis nesse processo, sem reações adversas significativas prejudiciais ao indivíduo em tratamento. A revisão bibliográfica foi realizada através de pesquisas em base de dados, mostrando a ação da espécie vegetal G. cambogia como ferramenta de auxílio no tratamento da obesidade. Os estudos com a G. cambogia se mostraram eficientes, principalmente no combate a obesidade, foi evidenciado que a espécie vegetal, principalmente o ácido hidroxicítrico (HCA) presente em seu fruto é responsável por gerar perda de peso em curto prazo, e na ingestão alimentar o HCA apresenta bons resultados, reduzindo acentualmente o número de refeições.

Palavras-chave: Obesidade; Tratamento; Fitoterápico; Garcinia cambogia.

\section{ABSTRACT:}

Based on data from the World Health Organization (WHO), obesity is among the most worrying morbidities currently, since this may still be a precursor to other diseases. Its treatment is extremely important, so taking it into account, this work aims to verify through a bibliographical review the use of the plant species Garcinia cambogia, as a coadjuvant in the treatment of obesity. Obesity has treatments aimed at food re-education and physical activity practice as basic treatments, but G. cambogia presents as an important phytotherapic, still emphasizing its benefits for visible results in this process, without significant adverse reactions that are harmful to the body. individual being treated. The bibliographical review was carried out through database searches, showing the action of the plant species G. cambogia as an aid tool in the treatment of obesity. The studies with $G$. cambogia showed to be efficient, mainly in the fight against obesity, it was evidenced that the vegetal species, mainly the hidroxycitric acid (HCA) present in its fruit is responsible for generating weight loss in the short term, and in the food intake the HCA presents good results, significantly reducing the number of meals.

Key Words: obesity; Treatment; Phytotherapic; Garcinia cambogia. 


\section{INTRODUÇÃO}

A obesidade é considerada como sendo o excedente de tecido adiposo, no organismo do indivíduo, é uma doença crônica e precursora, direta ou não de outras patogenias como, problemas cardiovasculares, hipertensão e neoplasias (CABRERA e FILHO, 2001). Ainda, a obesidade é caracterizada como sendo uma doença multifatorial que, segundo presunções da OMS, atinge mais de 300 milhões de indivíduos adultos em todo o planeta, visto que a sua prevalência é maior para com o sexo feminino, estima-se que a obesidade atinge $12,5 \%$ da população feminina, adulta, brasileira (LORENZI et al, 2005).

Há um significativo crescimento da prevalência da obesidade populacional em nível mundial, inclusive no Brasil. O ambiente vigente é um forte incentivo para o aumento da obesidade, visto que estamos frente à diminuição de atividade física, aumento da ingestão calórica alimentícia, além da mudança de estilo de vida, ou seja, da rotina presente na vida desses indivíduos, logo estes são fatores determinantes capazes de não apenas aumentar o desenvolvimento da obesidade, mas concretizá-la multifatorial (GODOY-MATOS et al, 2009).

O tratamento da obesidade é em geral nutricional, baseando-se em um planejamento de reeducação alimentar, associada à prática de atividade física, tornando ambos responsáveis diretos nos resultados positivo no tratamento. É significativa a utilização da fitoterapia como sendo um auxílio para esse tratamento auxiliar, considerando que há muitos anos os fitoterápicos estão presentes nos mais diversos tratamentos, devido às influências da cultura africana, indígena e europeia presentes no Brasil (PRADO et al, 2010).

A fitoterapia mostra-se como sendo uma excelente opção nos tratamentos atuais, em destaque para a obesidade. Esta alternativa apresenta-se trazendo melhorias no atendimento à população, a fim de proporcionar outro parâmetro auxiliar de tratamento, eficaz e com custos menores (BRUNING, MOSEGUI e VIANNA, 2012). Essa prática de prescrição fitoterápica é uma estratégia de complemento à prescrição terapêutica em envolvimento, visando à melhora da qualidade de vida dos pacientes, adesão ao tratamento e resultados positivos através da intervenção (OLIVEIRA e CORDEIRO, 2013).

A espécie vegetal Garcinia cambogia em seus estudos tem demonstrado um efeito positivo como uma ferramenta alternativa e coadjuvante para o tratamento da obesidade (ROSA e MACHADO, 2016). A G. cambogia, atua como inibidora de apetite e age 
bloqueando a absorção, além da síntese de gordura (SIMÃO, 2013), auxiliando na redução de peso, assim como $\theta$ nos níveis de LDL, colesterol total, e triglicérides do individuo (CORRÊA, SANTOS e RIBEIRO, 2012).

O extrato da G. cambogia é proveniente do pericarpo do seu fruto, e seu principal constituinte químico é o ácido hidroxicítrico ( $\mathrm{HCA}$ ), inibidor competitivo, ou seja, bloqueador da enzima extra mitocondrial citrato-liase. A função dessa enzima é a catálise/clivagem do citrato em acetilCoA e oxaloacetato, quando, bloqueada não ocorre a síntese lipídica, logo esse é um processo

fundamental para a redução de gordura do indivíduo (SANTOS et al, 2014).

Diante desse contexto, a adesão a produtos auxiliadores na redução e/ou manutenção do peso corpóreo dos indivíduos vem aumentando, sendo utilizado por uma grande parcela da população em todo mundo. Portanto, o objetivo deste trabalho foi verificar por meio de uma revisão bibliográfica a ação da espécie vegetal G. cambogia como coadjuvante no tratamento da obesidade, visando o bem-estar e a melhora dos indivíduos em tratamento.

\section{METODOLOGIA}

Esta pesquisa seguiu os princípios de uma revisão bibliográfica, isto é, levantamento de artigos científicos sobre o conteúdo relatado neste trabalho. Afim de uma integração literária com conteúdo específico e fundamentado sobre o tema.

Para isso, utilizaram-se as seguintes bases de dados: Google Acadêmico, SciELO e PubMed, com restrições de tempo, artigos de até 20 anos e os artigos pesquisados foram, na língua portuguesa e inglesa, utilizando os seguintes descritores de saúde: Garcinia cambogia, obesidade e fitoterapia.

\section{REFERENCIAL TEÓRICO}

\subsection{OBESIDADE}

Segundo dados da OMS, a obesidade é considerada nos dias atuais como sendo um dos problemas mais graves de saúde pública-em nível mundial (OMS, 2003). Ainda, a OMS estima a partir de projeções nacionais realizadas nas ultimas décadas, que a obesidade atingirá $30 \%$ da população na Inglaterra, $40 \%$ nos EUA e $20 \%$ no Brasil, dados 
projetados para o ano de 2025, salientando que pelo menos 1 bilhão de indivíduos apresentem excesso de peso, sendo desses, 300 milhões obesos (CONDE e BORGES, 2011).

A obesidade, além de ser considerada como uma epidemia mundial apresenta-se como sendo a mais importante desordem nutricional, tanto em países desenvolvidos como em países em desenvolvimento (RADAELLI, PEDROSO e MEDEIROS, 2016). Corresponde ainda, a um dos fatores de risco responsáveis pela prevalência de óbitos, além de estar associada a possíveis doenças (COUTINHO, GENTIL e TORAL, 2008). Essa patologia é de origem multifatorial, o direcionamento para revertê-la deve ser de forma indispensável, multidisciplinar. O seu tratamento é comumente direcionado à dieta (reeducação alimentar), ao exercício físico, a psicoterapia e, por vezes a recorrência por drogas anorexígenas (PINHEIRO, FREITAS e CORSO, 2004).

A introdução de aspectos direcionados a uma alimentação saudável é fundamental no processo do emagrecimento, recomenda-se que a ingestão energética seja balanceada. Pesquisas recentemente realizada nos EUA, mostram que o maior percentual deve ser dado ao total obtido de energia pelo indivíduo, em vez do total de gordura na dieta, logo o Ministério da Saúde do Brasil passou a disponibilizar Guias de Alimentação Saudável para a população, com o objetivo de alertar e incentivar a prática dessa mudança alimentar (ANJOS, 2006).

O gasto energético total de um indivíduo, por sua vez, se da de três formas: a taxa metabólica de repouso, sendo a energia necessária para que o organismo realize suas funções básicas, alcançando a homeostase, o efeito térmico dos alimentos, que é o gasto de energia para que ocorra a digestão, absorção, sintetização e estocagem de nutrientes no organismo e por fim, o gasto energético relacionado à atividade física, esse é dependente de movimentos voluntários e o gasto energético passa a ser variado, de indivíduo para indivíduo, mas é a forma mais facilmente modulável e intencional para a perda de gordura corporal (RIBEIRO e GARCIA, 2001).

Uma das formas classificatórias para a obesidade é o índice de massa corporal (IMC), em que ocorre a divisão do peso em quilos $(\mathrm{Kg})$, pelo quadrado da altura, medida em metros, (kg/m2). De acordo com a (OMS, 1998), a seguinte classificação foi adotada, representada pela tabela abaixo: 
TABELA 1 - Classificação da Obesidade Segundo o Índice de Massa Corporal (IMC)

\begin{tabular}{cc}
\hline Classificação & IMC $\mathbf{( k g / m 2 )}$ \\
\hline Sobrepeso & $\geq 25 \mathrm{e}<29,9$ \\
Obesidade grau 1 & $\geq 30 \mathrm{e}<34,9$ \\
Obesidade grau 2 & $\geq 35 \mathrm{e}<39,9$ \\
Obesidade grau 3 & $\geq 40$ \\
(anteriormente denominada obesidade mórbida). \\
\hline
\end{tabular}

Fonte: OMS, 1998

No entanto, essa classificação, avalia apenas o peso do indivíduo e não a sua composição corporal, sendo incapaz de identificar a taxa de gordura do mesmo, ou seja, o IMC não deve ser apenas o único parâmetro determinante para a classificação da obesidade (BORGES, BORGES, e SANTOS, 2006).

Segundo estudos, a obesidade apresenta maior predominância no sexo feminino, presume-se que aproximadamente $30 \%$ das mulheres adultas, próximas do estágio da menopausa, são portadoras dessa patologia. No Brasil, esse índice chega a 12,5\%. Essa condição apresenta gravidade maior às autoridades sanitárias mundiais, pois apresenta correlação direta com o desenvolvimento de doenças cardiovasculares, hipertensão arterial, diabetes mellitus, neoplasias, como o câncer de mama, cólon e endométrio (LORENZl et al, 2005).

Ainda, existem outras preocupações relacionadas á obesidade, menos discutidas, porém com grande relevância, em que incluem os aspectos psicológicos dos indivíduos associados à patologia, como, por exemplo, quando relacionados à imagem corporal, que por questões culturais é passível de se classificar ou criar estereótipos, logo qualquer outra condição fora desses aspectos passa a ter características negativas, favorecendo dessa forma, a discriminação e atos de insatisfação do próprio indivíduo para consigo mesmo (ALMEIDA et al, 2005).

\subsection{FITOTERAPIA}

Segundo a Portaria $n^{\circ} 6$ de 31 de janeiro de 1995, produto fitoterápico "É todo 
medicamento tecnicamente obtido e elaborado, empregando-se exclusivamente matériasprimas ativas vegetais com finalidade profilática, curativa ou para fins de diagnósticos, com benefício para o usuário". A mais recente RDC n²6, de 13 de maio de 2014 de fitoterápicos denomina esses como: "São considerados medicamentos fitoterápicos, os obtidos com emprego exclusivo de matérias-primas ativas vegetais, cuja segurança e eficácia sejam baseadas em evidências clínicas e que sejam caracterizados pela constância de sua qualidade".

O principal órgão responsável pela regulamentação de plantas medicinais e seus derivados no Brasil é a Agência Nacional de Vigilância Sanitária (ANVISA) (CARVALHO et al, 2008). Verifica-se um crescimento significativo na utilização de produtos fitoterápicos pela população brasileira, esse se da por dois motivos: Os avanços referentes à área científica, que permitem o desenvolvimento de fitoterápicos seguros e eficazes, e a crescente tendência, por parte da população, por uma terapêutica menos agressiva e/ou invasiva, destinada ao atendimento para com a saúde primária (BRUNING, MOSEGUI e VIANNA, 2012).

Quando ocorre a utilização de plantas medicinais de forma adequada essa mostrase de acordo com as proposições da OMS, que vem incentivado a utilização, bem como a valorização das terapias tradicionais (PRADO et al, 2010). Assim sendo, a fitoterapia compõe uma das alternativas disponíveis na "medicina alternativa", podendo atuar como coadjuvante nos mais diversos tratamentos. Entretanto, é de suma importância o domínio do conhecimento por parte dos profissionais atuantes na área de saúde, que estarão envolvidos diretamente na terapia juntamente aos pacientes (BRUNING, MOSEGUI e VIANNA, 2012).

Segundo Radaelli, Pedroso e Medeiros (2016) sob prescrição e acompanhamento de especialistas, conjunta à orientação farmacêutica, os medicamentos fitoterápicos podem ser utilizados no tratamento da obesidade, com o objetivo de resultar em melhorias no bemestar e da saúde metabólica do indivíduo.

Os fitoterápicos com ação emagrecedora agem no organismo com a finalidade de ação supressora de apetite, moderadores de ingestão alimentar, aceleradores de metabolismo, diminuindo ainda os níveis de colesterol, redutores de medida, além de atividade antioxidante, diurética e lipolítica (PELIZZA, 2010).

Este trabalho, aborda os benefícios oriundos dos fitoterápicos, em especial as vantagens na utilização da planta medicinal G. cambogia, visando seu auxílio no tratamento da obesidade, a fim de relatar evidências que sustentem o uso da G. cambogia como 
coadjuvante nesse processo (SANTOS et al, 2014).

\subsection{Garcínia cambogia (Gaertn.) Desr.}

A Garcínia é uma das possibilidades de escolha quando trata-se de plantas anorexígenas, é um gênero oriundo da Família Clusiaceae, possui diversas espécies de árvores e arbustos originária das Américas, Polinésia, África, e Ásia. Dentre suas espécies encontra-se a Garcínia cambogia, popularmente conhecida como Garcínia (MOUSINHO et al, 2014). Inúmeras dessas espécies também são encontradas facilmente na Índia, inclusive a G. cambogia, planta exótica nativa do Sul desse país. Na Índia a Garcínia é usada para o tratamento de diversas doenças e apresenta-se também para a finalidade de tempero, oferecendo sabores distintos aos pratos indianos (RADAELLI, PEDROSO e MEDEIROS, 2016). Antigamente a G. cambogia era utilizada com a função de conservante e aromatizante (PESSOA e SOUSA, 2017).

As partes utilizadas da espécie G. cambogia são casca seca e a polpa do fruto e possuem como principais metabolitos secundários os flavonoides, alcaloides, saponinas, compostos fenólicos, taninos, carboidratos e proteínas (TEIXEIRA, 2016). Ainda é constituída de antocianosídeos, lactonas hidroxicítricas, compostos fenólicos, pectina na polpa e sais minerais na casca e como corante, os demais compostos já isolados da planta estão descritos no quadro 1:

QUADRO 1 - Substâncias químicas presentes na G. cambogia

\begin{tabular}{|c|l|}
\hline CLASSE DE METABÓLITOS & \multicolumn{1}{|c|}{ SUBSTÂNCIAS QUíMICAS } \\
\hline BENZOFENONAS & $\begin{array}{l}\text { Garcinol, isogarcinol, guttiferone I, guttiferone } \\
\text { N, guttiferone J, guttiferone K e guttiferone M, } \\
\text { poliisopreniladas. }\end{array}$ \\
\hline XANTONAS & $\begin{array}{l}\text { Garbogiol, rheediaxantona A, xantonas } \\
\text { tetracíclicas poliisoprenilada (oxi-guttiferone } \\
\text { I, oxi-guttiferone K, oxi-guttiferone K2 e oxi- } \\
\text { guttiferone H). }\end{array}$ \\
\hline ÁcIDOS ORGÂNICOS & $\begin{array}{l}\text { Ácido Hidroxicítrico, ácido tartárico, ácido } \\
\text { cítrico e ácido málico. }\end{array}$ \\
\hline
\end{tabular}

FONTE: (MONTEIRO, 2008; TEIXEIRA, 2016). 
Segundo Radaelli, Pedroso e Medeiros (2016) o extrato da G. cambogia é obtido de seu fruto, mais especificamente do pericarpo. O principal componente químico é o ácido hidroxicítrico (HCA) (FIGURA 1). Atribuindo a essa planta a sua capacidade de controle e redução do peso corporal. O HCA apresenta ação inibitória do ATP citrato liase, bloqueando a clivagem do citrato em oxalato e acetil-CoA, procedimento indispensável para a síntese dos lipídios a partir de "oses", subunidades conduzidas para a formação de glicogênio, ainda essa inibição de acetil-CoA promove o aumento da oxidação dos ácidos graxos, ocasionando a produção de quantidade significativas de cetonas, que atuam na diminuição da deposição de gordura a partir da lipogênese (síntese de ácidos graxos e triglicérides armazenados no fígado e no tecido adiposo) (MOUSINHO et al, 2014). O HCA atua ainda de forma direta sobre os adipócitos, causando dispersão de gotículas lipídicas e alterações à transcrição (PESSOA e SOUSA, 2017).<smiles>O=C(O)CC(O)(C(=O)O)C(O)C(=O)O</smiles>

Ácido Hidroxicítrico<smiles>O=C(O)CC(O)(CC(=O)O)C(=O)O</smiles>

Ácido Cítrico<smiles>O=C(O)C(O)C(O)C(=O)O</smiles>

Ácido Tartárico<smiles>O=C(O)CC(O)C(=O)O</smiles>

Ácido Málico

\section{FIGURA 1 - ÁCIDOS ORGÂNICOS PRESENTES NO FRUTO G. cambogia}

Para Simão (2013) sua ação também se da pela redução do apetite, ou seja, controle também gerado a partir de uma maior síntese de glicogênio realizada pelo HCA, que difunde as calorias não armazenadas como gordura para geração de glicogênio, assim o cérebro recebe o sinal de saciedade, (através de receptores de açúcares atuantes no fígado). Ainda este processo possui a capacidade de aumentar a liberação de um 
neurotransmissor chamado serotonina, intimamente relacionado com o controle do apetite.

O HCA não tem a possibilidade de atravessar a barreira hematoencefálica, ou seja, não causa irregularidades ao Sistema Nervoso Central (SNC). A administração crônica do HCA na dosagem de 500mg (antes das refeições) promove o aceleramento do metabolismo energético, e como consequência a diminuição do peso nos indivíduos obesos (RADAELLI, PEDROSO e MEDEIROS, 2016; PESSOA e SOUSA, 2017).

Outros bioativos presentes na $G$. cambogia apresentam papel fundamental, dentre eles as benzofenonas, responsáveis por reduzir os níveis de estresse oxidativo, ou seja, a G. cambogia pode ser utilizada inclusive contra doenças relacionadas a está classe, como as doenças cardíacas (PESSOA e SOUSA, 2017).

Informações referentes à contraindicação na utilização da $G$. cambogia são desconhecidas (a partir da administração correta da mesma 1000mg/dia), bem como, seus efeitos secundários ou toxicidade (MONTEIRO, 2008). Com exceções vista a sua dosagem indicada, essa pode promover distúrbios gástricos (leves) e não deve ser utilizada para tratamento de gestantes, e em crianças menores de 12 anos. O efeito máximo obtido da $G$. cambogia é quando utilizada de 30 a 60 minutos previamente antes das refeições, visto que, sua utilização em doses fracionadas possui eficácia maior se comparado a sua administração em dose única (RADAELLI, PEDROSO e MEDEIROS, 2016).

Segundo Onakpoya et al (2010) foi evidenciado que a G. cambogia, principalmente o HCA presente em seu fruto, é responsável por gerar perda de peso em curto prazo. Quando relacionado à ingestão alimentar o HCA mostra-se bastante eficaz, reduzindo o número de refeições (CHUAH et al, 2013).

A G. cambogia ganha destaque, portanto na redução de ganho de peso, quando avaliada com indivíduos tratados com seu extrato, portanto conclui-se que a $G$. cambogia pode contribuir de forma significativa como coadjuvante para o tratamento da obesidade (VERRENGIA, KINOSHITA e AMADEI, 2013).

\section{CONCLUSÃO}

Nos países desenvolvidos e em desenvolvimento, inclusive o Brasil, desde a segunda metade das décadas de 70/80, houve um significativo crescimento das chamadas "medicinas alternativas", entre elas destaca-se a fitoterapia. Vemos, portanto que os fitoterápicos, mais especificamente a $G$. cambogia, são alternativas de tratamento que podem ser prescritos por médicos. Os estudos com a espécie mostram atividades 
principalmente no combate a obesidade, entretanto ainda são necessários mais estudos relacionados ao seu mecanismo de ação, procedimentos que esclareçam sua funcionalidade no organismo, o que promoveria uma maior segurança quando relacionado ao seu uso terapêutico, tanto por parte do prescritor quanto do próprio usuário. Os estudos, portanto, relatam a tendência e seu efeito positivo para o auxílio no tratamento e controle da obesidade, além de comorbidades em geral, ainda apresentando mínima possibilidade de reações adversas aos pacientes em tratamento.

\section{REFERÊNCIAS}

ALMEIDA, G. A. N. de et al. PERCEPÇÃO DE TAMANHO E FORMA CORPORAL DE MULHERES: ESTUDO EXPLORATÓRIO. Psicologia em Estudo, Maringá, v. 10, n. 1, p.27-35, jan /abr 2005.

ANVISA, Portaria $n^{\circ}$ 6, 31 de janeiro de 1995, produto fitoterápico. LEGISLAÇÃO RESOLUÇÃO DA DIRETORIA COLEGIADA - RDC N 26, DE 13 DE MAIO DE 2014. Ministério da Saúde Agência Nacional de Vigilância Sanitária; 13 maio 2014.

ANJOS, L. A. Prevenção e controle. In: Obesidade e saúde pública - online. Rio de Janeiro: Edit: FIOCRUZ, 2006. Temas em saúde collection, pp. 69-92.

BRUNING, M. C. R; MOSEGUI, G. B. G; VIANNA, C. M. de M. A utilização da fitoterapia e de plantas medicinais em unidades básicas de saúde nos municípios de Cascavel e Foz do Iguaçu - Paraná: a visão dos profissionais de saúde. Ciência \& Saúde Coletiva, Cascavel Pr., p.10, 2012.

CABRERA, M. A. S; FILHO, W. J; Obesidade em Idosos: Prevalência, Distribuição e Associação com Hábitos e Co-morbidades. Arq Bras Endocrinol Metab, v. 45 n 5 Outubro 2001.

CARVALHO, A. C. B. et al. Situação do registro de medicamentos fitoterápicos no Brasil. Revista Brasileira de Farmacognosia, Brasília-DF, Brasil, v. 18, n. 2, p.314-319, jan. 2008. 
CHUAH L. O. et al. Updates on Antiobesity Effect of Garcinia Origin (-)-HCA, EvidenceBased Complementary and Alternative Medicine, USA, 2013 Aug 6

CONDE, W. L; BORGES, C. O risco de incidência e persistência da obesidade entre adultos brasileiros segundo seu estado nutricional ao final da adolescência. Rev Bras Epidemiol, São Paulo (SP), Brasil, v. 1, n. 14, p.71-79, 10 jun, 2011.

CORRÊA, E. C. M; SANTOS, J. M. dos; RIBEIRO, P. L. B. USO DE FITOTERÁPICOS NO TRATAMENTO DA OBESIDADE UMA REVISÃO DE LITERATURA. TCC (Graduação) Curso de Especialista em Nutrição e Clínica Esportiva, Pontifícia Universidade Católica de Goiás, Goiânia, 2012.

COUTINHO, J. G; GENTIL, P. C; TORAL, N. A desnutrição e obesidade no Brasil: o enfrentamento com base na agenda única da nutrição. Cad. Saúde Pública. Rio de Janeiro, 03 jun. 2008. p. 5332-5340.

GODOY-MATOS, A. F. et al. Associação Brasileira para o Estudo da Obesidade e da Síndrome Metabólica Diretrizes brasileiras de obesidade 2009/2010 / ABESO Associação Brasileira para o Estudo da Obesidade e da Síndrome Metabólica; 3.ed, Itapevi, SP:AC Farmacêutica, 2009.

LORENZI, D. R. S. de et al. Prevalência de sobrepeso e obesidade no climatério: Prevalence of overweight and obesity among climacteric women. Rev Bras Ginecol Obstet, Caxias do Sul (RS), Brasil., p.479-484, 06 set. 2005.

MONTEIRO, A. R. M. Produtos à base de plantas dispensados em ervanárias para o emagrecimento: efeitos terapêuticos, toxicologia e legislação. Dissertação (Mestrado) - Curso de Medicina Legal, Instituto de Ciências Biomédicas de Abel Salazar da Universidade do Porto. Porto, 2008.

MOUSINHO, L. et al. Toxicidade de compostos químicos com ação anorexígena extraídos de Garcinia cambogia (Garcínia) e Panax ginseng (Ginseng). Revista Cubana de Plantas Medicinales, Picos, Piauí, Brasil, v. 19, n. 1, p.280-291, out. 2014. 
NONINO-BORGES, C. B; BORGES, R. M; SANTOS, J. E. dos. TRATAMENTO CLÍNICO DA OBESIDADE. TCC (Graduação) - Curso de Medicina, Centro Universitário Barão de Mauá, Ribeirão Preto, 2006. Cap. 39.

OLIVEIRA, I. C; CORDEIRO, P. B. de M. H. Os Fitoterápicos como Coadjuvantes no Tratamento da Obesidade. Cadernos UniFOA, Edição Especial do Curso de Nutrição, RJ, Brasil - maio, 2013.

ONAKPOYA, I. et al. The Use of Garcinia Extract (Hydroxycitric Acid) as a Weight loss Supplement: A Systematic Review and Meta-Analysis of Randomised Clinical Trials. Journal Of Obesity, Reino Unido. p.1-17, 10 dez. 2010.

PELIZZA, M. C. Uso de Cereus sp. e Cordia ecalyculata Vell como emagrecedores: uma revisão. TCC (Graduação) - Curso de Farmácia, Faculdade, Universidade Federal do Rio Grande do Sul, Porto Alegre, 2010.

PESSOA, É. V. M; SOUSA, F. das C. A. Effect of garcinia's administration changes in weight reduction. Reonfacema, Maranhão, v.3, n.2, p.513-518, abr-jun. 2017.

PINHEIRO, A. R. de O; FREITAS, S. F. T. de; CORSO, A. C. T. Uma abordagem epidemiológica da obesidade. Revista de Nutrição, Campinas, v. 17(4), p.523-533, 16 out. 2003.

PRADO, C. $\mathrm{N}$. do et al. O USO DE FITOTERÁPICOS NO TRATAMENTO DA OBESIDADE. Revista Brasileira de Obesidade, Nutrição e Emagrecimento: Periódico do Instituto Brasileiro de Pesquisa e Ensino em Fisiologia do Exercício, São Paulo, v. 4, p.14-21, jan. 2010.

RADAELLI, M; PEDROSO, R. C; MEDEIROS, L. F. Farmacoterapia da obesidade: Benefícios e Riscos. Revista Saúde e Desenvolvimento Humano - Issn 23178582, Canoas, v. 4, n. 1, p.101-115, abr, 2016.

RIBEIRO, E. A. G; GARCIA L. M. T. Atividade física e obesidade, publicado em: ResearchGate, Santa Catarina, 07 jun, 2016. 
ROSA, F. M. M; MACHADO, J. T. O efeito anti-obesidade da Garcinia cambogia em humanos. Revista Fitos, [s.I.], v. 10, n. 2, p.95-219, 2016.

SANTOS, A. C. S, et al. Garcinia cambogia - uma espécie vegetal como recurso terapêutico contra a obesidade? Natureza On Line, Bairro Dois Pinheiros, Santa Teresa, ES, Brasil, v. 5, n. 1, p.37-43, 13 mar, 2014.

SIMÃO, A. A. Composição química, eficácia e toxicidade de plantas medicinais utilizadas no tratamento da obesidade. Tese (Doutorado) - Curso de Agroquímica, Universidade Federal de Lavras, Lavras - MG, 2013.

TEIXEIRA, M. S. M. AVALIAÇÃO DA ATIVIDADE FOTOPROTETORA DE FORMULAÇÃO COSMÉTICA CONTENDO A ASSOCIAÇÃO ENTRE FRAÇÃO EM CLOROFÓRMIO DE Garcinia cambogia Desr. (Clusiaceae) E FILTRO SINTÉTICO DE AMPLO ESPECTRO. Pág. 61, TCC (Graduação) - Curso de Farmácia, Universidade Federal de Juiz de Fora, Juiz de Fora, 2016.

VERRENGIA, E. C; KINOSHITA, S. A. T; AMADEI, J. L. Medicamentos Fitoterápicos no Tratamento da Obesidade. UNICIÊNCIAS, v.17, n.1, p.53-58, dez. 2013. 